\title{
Replication of Mini-Sentinel Study Assessing Mirabegron and Cardiovascular Risk in Non-Mini-Sentinel Databases
}

\author{
Jason C. Simeone ${ }^{1} \cdot$ Beth L. Nordstrom $^{1} \cdot$ Kwame Appenteng $^{2}$. \\ Samuel Huse ${ }^{1} \cdot$ Milbhor D'Silva $^{2}$
}

Published online: 13 November 2017

(c) The Author(s) 2017. This article is an open access publication

\begin{abstract}
Background In 2014, the US Food and Drug Administration (FDA) initiated a prospective routine surveillance using the Mini-Sentinel (M-S) program to assess potential signals of acute myocardial infarction (AMI) and stroke with use of mirabegron, indicated for the treatment of overactive bladder (OAB), compared with oxybutynin.

Purpose To replicate the FDA M-S analysis of mirabegron using datasets that did not contribute to the M-S program. Methods IMS PharMetrics Plus and Truven MarketScan claims data from 2012-2015 were converted to the M-S Common Data Model. New and non-new users of mirabegron and oxybutynin were analyzed per the publicly available M-S protocol, and propensity score-matched 1:1 using the M-S PROMPT 2 module. Incidence rates (IR) were calculated per 1000 person-years (PY). Adjusted hazard ratios (aHRs) for mirabegron versus oxybutynin were calculated using Cox regression models.
\end{abstract}

Jason C. Simeone

jason.simeone@evidera.com

Beth L. Nordstrom

beth.nordstrom@evidera.com

Kwame Appenteng

kwame.appenteng@astellas.com

Samuel Huse

samuel.huse@evidera.com

Milbhor D'Silva

milbhor.d'silva@astellas.com

1 Real-World Evidence, Evidera, 500 Totten Pond Road, Fifth Floor, Waltham, MA 02451, USA

2 Safety Science, Astellas Pharma US, Inc., 1 Astellas Way, Northbrook, IL 60062, USA
Results In PharMetrics, 12,429 new mirabegron users and 61,548 new oxybutynin users were identified. The aHR was 0.67 (95\% confidence interval (CI)] 0.33-1.37) for AMI (mirabegron IR 4.4/1000 PY), and 0.62 (95\% CI 0.34-1.13) for stroke (mirabegron IR 6.3/1000 PY). In MarketScan, 17,182 new mirabegron users and 63,962 new oxybutynin users were identified. The aHR was 0.57 (95\% CI 0.17-1.95) for AMI, and 0.69 (95\% CI 0.30-1.62) for stroke; IRs were similar to those from PharMetrics. Neither dataset suggested an increased risk of AMI or stroke associated with mirabegron in non-new users.

Conclusions Using the publicly-available M-S protocol and analysis programs with alternative (non M-S) data sources, no statistically significant increased risk of AMI or stroke was found among new or non-new users of mirabegron compared with oxybutynin. These findings were consistent with the FDA M-S mirabegron study. 


\section{Key Points}

The Mini-Sentinel safety study of mirabegron (a treatment for overactive bladder) was replicated using the publicly-available Mini-Sentinel common data model specifications, protocol, and analysis modules using IMS PharMetrics and Truven MarketScan, two databases that do not contribute to Mini-Sentinel.

Propensity score-matched Cox proportional hazards models indicated no increased risk of acute myocardial infarction or stroke among either new or non-new users of mirabegron compared to oxybutynin in either dataset.

Findings from the present study are consistent with the results of the US Food and Drug Administration's Mini-Sentinel report on mirabegron, and the methods described here could be considered for other therapeutic areas, drugs, and outcomes of interest.

\section{Introduction}

Overactive bladder (OAB) was defined in 2002 by the International Continence Society as a condition characterized by urgency with or without incontinence, generally in the presence of frequency and nocturia, and suggestive of lower urinary tract dysfunction [1-3]. OAB is a common disorder and occurs in a wide range of patients, from the young to the very elderly. $\mathrm{OAB}$ increases with age in both sexes, and it is often underdiagnosed and undertreated. The main symptom of $\mathrm{OAB}$ is urgency, and, therefore, persons with this symptom are considered to have $\mathrm{OAB}$. The symptoms of $\mathrm{OAB}$, particularly urinary urgency and urinary incontinence, can have a considerable impact on quality of life [4].

Mirabegron is a beta-3 adrenergic agonist indicated for the treatment of $\mathrm{OAB}$ with symptoms of urge urinary incontinence, urgency, and urinary frequency. During clinical development, mirabegron at a dose of $50 \mathrm{mg}$ once daily was associated with mean increases in pulse rate of approximately one beat per minute compared with placebo, and a mean increase in blood pressure (BP) of $0.5-1 \mathrm{mmHg}$ (systolic and diastolic) compared with placebo in patients with OAB [5].

In population-based epidemiologic studies, increased levels of heart rate and BP have been positively associated with the risk of stroke and coronary heart disease (CHD) [6]. Randomized trials have shown that pharmacologically reducing diastolic blood pressure by $5-6 \mathrm{mmHg}$ for a few years in hypertensive patients was associated with relative reductions in stroke and CHD risk of 42 and 14\%, respectively [6]. A $5 \mathrm{mmHg}$ reduction in systolic BP resulted in a $14 \%$ overall reduction in mortality due to stroke and a $9 \%$ reduction in mortality due to CHD in hypertensive $(\geq 140 / 90 \mathrm{mmHg}$ ) patients [7].

In June 2014, following the approval of mirabegron for the treatment of OAB, the US Food and Drug Administration (FDA) initiated a prospective routine observational surveillance assessment as part of the Mini-Sentinel (M-S) program to identify potential signals of acute myocardial infarction (AMI) and stroke with use of mirabegron. The objective of this research was to replicate the FDA's study, using databases not contributing to the M-S program.

\section{Methods}

The present study was a retrospective cohort study of US administrative claims data from the IMS PharMetrics Plus and Truven MarketScan databases from July 2012 to the latest date available in each database (June 2015 in MarketScan and September 2015 in PharMetrics). Mirabegron was approved by the FDA on 28 June 2012.

These databases were then converted to the FDA's M-S Common Data Model (CDM), using publicly-available specifications [8]. Conversion to the CDM permits the use of the M-S Prospective Routine Observational Monitoring Program Tool: Cohort Matching (PROMPT 2 module) [9]—an assessment tool using a propensity score (PS)matched cohort design. The use of this module was specified in the M-S protocol for mirabegron and generates analyses that can be easily compared to estimates from the FDA's M-S mirabegron safety study.

One minor CDM deviation was made to accommodate the IMS PharMetrics dataset. Per the FDA's M-S CDM specifications [8], a Provider ID is required to count distinct patient visits. As the IMS PharMetrics data does not include a Provider ID in the standard data available for licensing, visits to specific providers could not be directly identified. Therefore, multiple visits to the same provider in the same day were defined as visits in the same day and setting, with the same diagnosis codes across all fields; these were counted as one encounter for consistency with the CDM specifications. Visits on different days, visits on the same day in different settings, or visits on the same day with different diagnoses were considered to be separate encounters.

\subsection{Cohort Selection}

The index date was the first date of exposure to mirabegron or oxybutynin, and the baseline period was defined as the 
183-day period prior to and excluding the index date. The primary analysis included new mirabegron and oxybutynin users, and new users were defined as those without any $\mathrm{OAB}$ prescription during the baseline period. The secondary analysis included non-new users of the same drugs, where non-new use was defined as an initiation of the cohort-defining drug, with at least one exposure to another $\mathrm{OAB}$ drug other than the cohort-defining drug during the baseline period. Therefore, this non-new user analysis includes patients who initiated the cohort-defining drug with recent prior $\mathrm{OAB}$ drug use, but is not limited to patients who actively switched from one OAB drug to the cohort-defining drug at the index date.

Patients aged $<20$ years old and those newly-initiating mirabegron or oxybutynin on the same day as another OAB drug were excluded from the study. Persons with an AMI or stroke in the 30 days prior to the index date were excluded from the analysis of that respective outcome.

\subsection{Follow-up and Censoring}

Follow-up time began on the index date with the cohort entry-defining mirabegron or oxybutynin dispensing and continued based on the number of days supplied of prescriptions for these agents. Follow-up ended (i.e., persontime was censored) upon the earliest occurrence of: the outcome of interest, a gap of $\geq 7$ days between two consecutive prescriptions for the cohort-defining agent, discontinuation of the cohort-defining agent, a prescription for an $\mathrm{OAB}$ drug other than the cohort-defining agent, end of the study period, or health plan disenrollment. The earliest censoring event occurring for either person in a matched pair served as the censoring date for both persons in the pair.

AMI, the primary outcome of interest, was defined by an International Classification of Diseases, 9th Revision, Clinical Modification (ICD-9-CM) inpatient diagnosis of 410.X0 or 410.X1 in the principal position on an inpatient record. Stroke, the secondary outcome of interest, was identified by the presence of an ICD-9-CM code of 430, $431,433 . X 1,434 . X 1$, or 436 in the principal position on an inpatient record.

The PROMPT 2 module controls for confounding by generating PS from the pre-defined lists of covariates specified and defined in the M-S protocol; all covariate definitions used in the present study match those specified in the M-S protocol [10]. These lists include baseline variables related to demographic characteristics (e.g., age, sex), healthcare resource utilization (e.g., number of visits), and clinical characteristics (e.g., co-morbidities and medication use). All covariates listed were identified using information available in claims databases, such as diagnosis and procedure codes.

\subsection{Statistical Analysis}

Statistical analyses were conducted with $\mathrm{SAS}^{\circledR}$ 9.4, using the published PROMPT 2 module as described previously.

The PROMPT 2 module was used to match mirabegronexposed persons to oxybutynin-exposed persons by PS at a 1:1 ratio. This module implements the matching process outlined in the M-S PROMPT: Cohort Matching Technical Users' Guide [9]. Nearest neighbor matching on PS was conducted using a caliper distance of 0.025 units on the PS scale. Baseline characteristics of the unmatched and matched treatment cohorts and time on drug were summarized using descriptive statistics including means, standard deviations (SDs), medians, and ranges for continuous variables, and frequencies for categorical variables. Per the FDA's M-S protocol, the PROMPT 2 module does not generate data for the matched cohorts if the PS matching model does not converge (i.e., does not complete the PS identification and/or matching process). This may be due to covariates that occur infrequently, leading to very small cell sizes.

The PROMPT 2 module then conducts a Cox regression model and generates hazard ratios (HR) for mirabegron use compared to oxybutynin use with $95 \%$ confidence intervals (CIs), as well as other risk-associated information in the unmatched treatment cohorts. Adjusted hazard ratios (aHRs) and 95\% CIs were calculated from Cox regression models stratified by PS decile without trimming, and in the matched treatment cohorts, if the PS matching model converged. Incidence rates (IRs) were calculated per 1000 person-years (PY) using the number of outcomes and person time in the matched treatment groups when the model converged, or in the unmatched groups if the matching model did not converge. All PS deciles contained patients from each treatment cohort. Details on the analytic approach are specified in the PROMPT User's Guide [11]. Results are reported for each dataset, in both the primary (new users) and secondary (non-new users) analyses, and for both outcomes, for a total of eight sets of analyses.

\section{Results}

\subsection{IMS PharMetrics Plus Population}

\subsubsection{New Users}

For the AMI analysis, the mean age of 12,429 new mirabegron users was $55.6 \pm 12.3$ years, compared to $52.9 \pm 13.3$ years among 61,548 new oxybutynin users (Table 1). Among mirabegron users, $73.8 \%$ were women, compared to $64.0 \%$ of oxybutynin users. Mirabegron users had a lower mean number of healthcare visits when 
Table 1 Baseline characteristics of new and non-new users from IMS PharMetrics for the AMI outcome analysis

\begin{tabular}{|c|c|c|c|c|}
\hline Baseline patient characteristics & $\begin{array}{l}\text { Pre-matching } \\
\text { mirabegron } \\
\text { Mean }(\mathrm{SD})^{\mathrm{a}}\end{array}$ & $\begin{array}{l}\text { Pre-matching } \\
\text { oxybutynin } \\
\text { Mean }(\mathrm{SD})^{\mathrm{a}}\end{array}$ & $\begin{array}{l}\text { Post-matching } \\
\text { mirabegron } \\
\text { Mean }(\mathrm{SD})^{\mathrm{a}}\end{array}$ & $\begin{array}{l}\text { Post-matching } \\
\text { oxybutynin } \\
\text { Mean (SD) }\end{array}$ \\
\hline New users & $(N=12,429)$ & $(N=61,548)$ & NA & NA \\
\hline Age (years) at index date & $55.6(12.3)$ & $52.9(13.3)$ & NA & NA \\
\hline \multicolumn{5}{|l|}{ Gender $(N[\%])$} \\
\hline Male & $3260(26.2 \%)$ & $22,172(36.0 \%)$ & NA & NA \\
\hline Female & $9167(73.8 \%)$ & $39,376(64.0 \%)$ & NA & NA \\
\hline $\begin{array}{l}\text { Charlson-Elixhauser co-morbidity } \\
\text { score }\end{array}$ & $0.4(1.3)$ & $0.5(1.6)$ & NA & NA \\
\hline Inpatient hospitalizations & $0.6(3.6)$ & $1.3(5.8)$ & NA & NA \\
\hline Emergency department visits & $0.2(0.7)$ & $0.6(1.3)$ & NA & NA \\
\hline Non-acute institutional stays & $0.1(1.3)$ & $0.2(2.0)$ & NA & NA \\
\hline Ambulatory visits & $2.5(4.7)$ & $3.2(5.7)$ & NA & NA \\
\hline Other ambulatory events & $10.3(9.7)$ & $8.2(9.4)$ & NA & NA \\
\hline Unique prescriptions & $19.3(17.8)$ & $16.1(16.6)$ & NA & NA \\
\hline Unique generics & $8.0(5.8)$ & $7.0(5.5)$ & NA & NA \\
\hline Non-new users & $(N=9025)$ & $(N=7899)$ & $(N=5172)$ & $(N=5172)$ \\
\hline Age (years) at index date & $57.9(12.6)$ & $57.2(12.4)$ & $57.9(12.5)$ & $57.2(12.3)$ \\
\hline \multicolumn{5}{|l|}{ Gender $(N[\%])$} \\
\hline Male & $1996(22.1 \%)$ & $1719(21.8 \%)$ & $1138(22.0 \%)$ & $1134(21.9 \%)$ \\
\hline Female & $7029(77.9 \%)$ & $6179(78.2 \%)$ & $4034(78.0 \%)$ & $4038(78.1 \%)$ \\
\hline $\begin{array}{l}\text { Charlson-Elixhauser co-morbidity } \\
\text { score }\end{array}$ & $0.4(1.4)$ & $0.6(1.7)$ & $0.4(1.4)$ & $0.4(1.4)$ \\
\hline Inpatient hospitalizations & $0.6(3.5)$ & $1.5(6.7)$ & $0.8(4.4)$ & $0.7(3.6)$ \\
\hline Emergency department visits & $0.3(0.8)$ & $0.4(1.2)$ & $0.3(0.9)$ & $0.3(0.8)$ \\
\hline Non-acute institutional stays & $0.1(1.2)$ & $0.2(1.9)$ & $0.2(1.3)$ & $0.2(1.1)$ \\
\hline Ambulatory visits & $3.0(5.4)$ & $3.1(5.4)$ & $2.8(5.3)$ & $2.8(4.8)$ \\
\hline Other ambulatory events & $11.8(11.0)$ & $9.9(10.8)$ & $10.3(10.1)$ & $10.2(10.7)$ \\
\hline Unique prescriptions & $25.8(19.5)$ & $24.4(20.5)$ & $24.2(18.7)$ & $24.2(19.6)$ \\
\hline Unique generics & $10.2(5.9)$ & $9.6(6.0)$ & $9.6(5.6)$ & $9.6(6.0)$ \\
\hline
\end{tabular}

$A M I$ acute myocardial infarction, $N A$ not applicable (propensity score matching model did not converge), $S D$ standard deviation ${ }^{\mathrm{a}}$ Data appear as mean (SD) except for gender data, which appear as $N(\%)$

compared to oxybutynin users, including inpatient stays (0.6 vs. 1.3 ), emergency department visits ( 0.2 vs. 0.6 ), and ambulatory visits (2.5 vs. 3.2 ). Mirabegron users had a higher mean number of prescriptions when compared to oxybutynin users (19.3 vs. 16.1). Matched characteristics are not presented as the PS matching model did not converge.

\subsubsection{Non-new Users}

A total of 9025 non-new mirabegron users and 7899 nonnew oxybutynin users were identified for the AMI analysis. Mean ages (mirabegron 57.9 \pm 12.6 , oxybutynin $57.2 \pm 12.4$ ) were higher than for new users (see above), a higher proportion of non-new users (77.9\% of mirabegron users and $78.2 \%$ of oxybutynin users) were women compared to new users (see above), and non-new users had a higher mean number of prescriptions (mirabegron $25.8 \pm 19.5$, oxybutynin $24.4 \pm 20.5$ ). After PS-matching, 5172 patients remained in each cohort, and patient characteristics were similar across cohorts.

Characteristics of the new and non-new users identified for the stroke analysis are presented in Table 2. Among 12,379 new mirabegron users and 61,411 new oxybutynin users, characteristics were similar to new users identified for the AMI analysis, and the PS-matching model did not converge. Among non-new users, 8959 mirabegron users and 7872 oxybutynin users were identified with similar characteristics to non-new users in the AMI analysis; 5236 patients remained in each treatment cohort after PSmatching. 
Table 2 Baseline characteristics of new and non-new users from IMS PharMetrics for the stroke outcome analysis

\begin{tabular}{|c|c|c|c|c|}
\hline Baseline patient characteristics & $\begin{array}{l}\text { Pre-matching } \\
\text { mirabegron } \\
\text { Mean }(\mathrm{SD})^{\mathrm{a}}\end{array}$ & $\begin{array}{l}\text { Pre-matching } \\
\text { oxybutynin } \\
\text { Mean }(\mathrm{SD})^{\mathrm{a}}\end{array}$ & $\begin{array}{l}\text { Post-matching } \\
\text { mirabegron } \\
\text { Mean }(\mathrm{SD})^{\mathrm{a}}\end{array}$ & $\begin{array}{l}\text { Post-matching } \\
\text { oxybutynin } \\
\text { Mean (SD) }\end{array}$ \\
\hline New users & $(N=12,379)$ & $(N=61,411)$ & NA & NA \\
\hline Age at index date & $55.6(12.3)$ & $52.9(13.3)$ & NA & NA \\
\hline \multicolumn{5}{|l|}{ Gender $(N[\%])$} \\
\hline Male & $3235(26.1 \%)$ & $22,133(36.0 \%)$ & NA & NA \\
\hline Female & $9142(73.9 \%)$ & $39,278(64.0 \%)$ & NA & NA \\
\hline $\begin{array}{l}\text { Charlson-Elixhauser co-morbidity } \\
\text { score }\end{array}$ & $0.4(1.3)$ & $0.5(1.6)$ & NA & NA \\
\hline Inpatient hospitalizations & $0.5(3.1)$ & $1.2(5.6)$ & NA & NA \\
\hline Emergency department visits & $0.2(0.7)$ & $0.6(1.3)$ & NA & NA \\
\hline Non-acute institutional stays & $0.1(1.2)$ & $0.2(1.9)$ & NA & NA \\
\hline Ambulatory visits & $2.4(4.6)$ & $3.2(5.7)$ & NA & NA \\
\hline Other ambulatory events & $10.3(9.7)$ & $8.2(9.3)$ & NA & NA \\
\hline Unique prescriptions & $19.2(17.7)$ & $16.1(16.5)$ & NA & NA \\
\hline Unique generics & $8.0(5.7)$ & $7.0(5.5)$ & NA & NA \\
\hline Non-new users & $(N=8959)$ & $(N=7872)$ & $(N=5236)$ & $(N=5236)$ \\
\hline Age at index date & $57.9(12.5)$ & $57.2(12.4)$ & $58.0(12.5)$ & $57.0(12.3)$ \\
\hline \multicolumn{5}{|l|}{ Gender $(N[\%])$} \\
\hline Male & $1967(22.0 \%)$ & $1710(21.7 \%)$ & $1151(22.0 \%)$ & $1159(22.1 \%)$ \\
\hline Female & $6992(78.0 \%)$ & $6161(78.3 \%)$ & $4085(78.0 \%)$ & $4077(77.9 \%)$ \\
\hline $\begin{array}{l}\text { Charlson-Elixhauser co-morbidity } \\
\text { score }\end{array}$ & $0.4(1.4)$ & $0.6(1.7)$ & $0.4(1.4)$ & $0.4(1.4)$ \\
\hline Inpatient hospitalizations & $0.6(3.3)$ & $1.5(6.6)$ & $0.7(4.0)$ & $0.7(3.7)$ \\
\hline Emergency department visits & $0.2(0.7)$ & $0.4(1.2)$ & $0.3(0.8)$ & $0.3(0.8)$ \\
\hline Non-acute institutional stays & $0.1(1.1)$ & $0.2(1.9)$ & $0.2(1.3)$ & $0.1(1.0)$ \\
\hline Ambulatory visits & $2.9(5.3)$ & $3.1(5.3)$ & $2.9(5.7)$ & $2.8(4.9)$ \\
\hline Other ambulatory events & $11.8(11.0)$ & $9.9(10.8)$ & $10.1(9.8)$ & $10.3(10.7)$ \\
\hline Unique prescriptions & $25.7(19.4)$ & $24.3(20.4)$ & $24.2(18.2)$ & $24.3(19.9)$ \\
\hline Unique generics & $10.1(5.9)$ & $9.5(6.0)$ & $9.6(5.6)$ & $9.6(5.9)$ \\
\hline
\end{tabular}

$N A$ not applicable (propensity score matching model did not converge), $S D$ standard deviation

${ }^{a}$ Data appear as mean (SD) except for gender data, which appear as $N(\%)$

\subsection{Truven MarketScan Population}

\subsubsection{New Users}

The analysis of AMI from Truven MarketScan included 17,182 new mirabegron users and 63,962 new oxybutynin users (Table 3). The mean age of mirabegron users was $64.7 \pm 15.2$ years, compared to $59.7 \pm 16.2$ for oxybutynin users. Over two-thirds $(68.0 \%)$ of mirabegron users were women, compared to $63.2 \%$ of oxybutynin users. Mirabegron users generally had lower mean resource utilization than oxybutynin users, including inpatient visits ( 0.4 vs. $0.6)$, emergency department visits (0.2 vs. 0.4$)$, and ambulatory visits (2.4 vs. 2.9). Among mirabegron patients, the mean number of prescriptions received was $18.7 \pm 15.5$, compared to $16.3 \pm 15.0$ among oxybutynin patients. After matching, 16,452 patients remained in each cohort; patient characteristics in those cohorts were generally similar.

\subsubsection{Non-new Users}

The analysis of AMI in the non-new user cohort included 15,252 mirabegron users and 11,374 oxybutynin users; mean ages were higher (mirabegron $66.3 \pm 14.5$, oxybutynin $65.4 \pm 15.3$ ) than new users in MarketScan, and approximately $75 \%$ of both cohorts were women. The mean number of prescriptions (mirabegron $24.3 \pm 17.6$, oxybutynin $23.4 \pm 17.9$ ) was also higher than for new users, but other characteristics were similar. After matching, 8123 patients remained in each cohort with similar characteristics. 
Table 3 Baseline characteristics of new and non-new users from Truven MarketScan for the AMI outcome analysis

\begin{tabular}{|c|c|c|c|c|}
\hline Baseline patient characteristics & $\begin{array}{l}\text { Pre-matching } \\
\text { mirabegron } \\
\text { Mean }(\mathrm{SD})^{\mathrm{a}}\end{array}$ & $\begin{array}{l}\text { Pre-matching } \\
\text { oxybutynin } \\
\text { Mean }(\mathrm{SD})^{\mathrm{a}}\end{array}$ & $\begin{array}{l}\text { Post-matching } \\
\text { mirabegron } \\
\text { Mean (SD) }\end{array}$ & $\begin{array}{l}\text { Post-matching } \\
\text { oxybutynin } \\
\text { Mean (SD) }\end{array}$ \\
\hline New users & $(N=17,182)$ & $(N=63,962)$ & $(N=16,452)$ & $(N=16,452)$ \\
\hline Age at index date & $64.7(15.2)$ & $59.7(16.2)$ & $64.5(15.2)$ & $62.0(16.1)$ \\
\hline \multicolumn{5}{|l|}{ Gender $(N[\%])$} \\
\hline Male & $5501(32.0 \%)$ & $23,542(36.8 \%)$ & $5289(32.1 \%)$ & $5208(31.7 \%)$ \\
\hline Female & $11,681(68.0 \%)$ & $40,420(63.2 \%)$ & $11,163(67.9 \%)$ & $11,244(68.3 \%)$ \\
\hline $\begin{array}{l}\text { Charlson-Elixhauser co-morbidity } \\
\text { score }\end{array}$ & $0.6(1.5)$ & $0.7(1.8)$ & $0.6(1.5)$ & $0.6(1.6)$ \\
\hline Inpatient hospitalizations & $0.4(2.1)$ & $0.6(2.5)$ & $0.4(2.1)$ & $0.4(1.7)$ \\
\hline Emergency department visits & $0.2(0.6)$ & $0.4(0.9)$ & $0.2(0.7)$ & $0.2(0.6)$ \\
\hline Non-acute institutional stays & $0.2(2.3)$ & $0.3(3.6)$ & $0.2(2.3)$ & $0.3(2.1)$ \\
\hline Ambulatory visits & $2.4(4.3)$ & $2.9(5.0)$ & $2.4(4.3)$ & $2.3(4.0)$ \\
\hline Other ambulatory events & $11.5(10.0)$ & $8.6(9.1)$ & $11.1(9.6)$ & $11.2(11.4)$ \\
\hline Unique prescriptions & $18.7(15.5)$ & $16.3(15.0)$ & $18.5(15.5)$ & $18.6(15.7)$ \\
\hline Unique generics & $8.6(5.5)$ & $7.4(5.2)$ & $8.4(5.4)$ & $8.5(5.6)$ \\
\hline Non-new users & $(N=15,252)$ & $(N=11,374)$ & $(N=8123)$ & $(N=8123)$ \\
\hline Age at index date & $66.3(14.5)$ & $65.4(15.3)$ & $65.9(14.8)$ & $64.9(14.9)$ \\
\hline \multicolumn{5}{|l|}{ Gender $(N[\%])$} \\
\hline Male & $4104(26.9 \%)$ & $2727(24.0 \%)$ & $2045(25.2 \%)$ & $2034(25.0 \%)$ \\
\hline Female & $11,148(73.1 \%)$ & $8647(76.0 \%)$ & $6078(74.8 \%)$ & $6089(75.0 \%)$ \\
\hline $\begin{array}{l}\text { Charlson-Elixhauser co-morbidity } \\
\text { score }\end{array}$ & $0.7(1.6)$ & $0.8(2.0)$ & $0.7(1.7)$ & $0.7(1.7)$ \\
\hline Inpatient hospitalizations & $0.4(1.9)$ & $0.9(3.5)$ & $0.5(2.2)$ & $0.5(2.1)$ \\
\hline Emergency department visits & $0.3(0.7)$ & $0.4(0.9)$ & $0.3(0.8)$ & $0.3(0.7)$ \\
\hline Non-acute institutional stays & $0.3(2.6)$ & $0.4(2.9)$ & $0.3(2.7)$ & $0.3(2.5)$ \\
\hline Ambulatory visits & $2.8(5.1)$ & $2.9(4.9)$ & $2.7(5.2)$ & $2.7(4.6)$ \\
\hline Other ambulatory events & $12.2(10.5)$ & $10.4(10.2)$ & $10.7(9.8)$ & $10.6(10.0)$ \\
\hline Unique prescriptions & $24.3(17.6)$ & $23.4(17.9)$ & $23.5(17.2)$ & $23.5(17.7)$ \\
\hline Unique generics & $10.5(5.7)$ & $9.9(5.6)$ & $10.0(5.5)$ & $10.0(5.6)$ \\
\hline
\end{tabular}

$A M I$ acute myocardial infarction, $N A$ not applicable (propensity score matching model did not converge, $S D$ standard deviation

${ }^{a}$ Data appear as mean (SD) except for Gender data, which appear as $N(\%)$

A total of 17,138 new mirabegron users and 63,835 new oxybutynin users met the criteria for inclusion in the stroke outcome analysis (Table 4), with similar characteristics to the AMI analysis of new users in the same dataset; 15,973 patients remained in each cohort after matching. In the non-new user analysis of stroke, 15,173 mirabegron patients and 11,314 oxybutynin patients were selected, and their characteristics were again similar to the non-new users in the AMI analysis. After matching, 8103 patients in each cohort remained with similar characteristics.

\subsection{Outcomes}

Outcome analyses from new and non-new users in both datasets are presented in Table 5.
Among new users in the AMI outcome analysis in IMS PharMetrics, the mean length of drug exposure was 79 days on mirabegron and 44 days on oxybutynin (data not shown). The IR of AMI was 4.4/1000 PY for mirabegron and 6.5/1000 PY for oxybutynin. Prior to matching, the HR for AMI was 0.68 (95\% CI 0.36-1.28), similar to the aHR after PS decile stratification $(0.67 ; 95 \%$ CI 0.33-1.37). The PS-matched model failed to converge.

Among non-new users in the PharMetrics AMI analysis, the IR for mirabegron users was 5.8/1000 PY compared to 2.7/1000 PY among oxybutynin users. Point estimates varied, but no statistically significant association between mirabegron use and AMI was observed for unmatched users (HR 0.95; 95\% CI 0.38-2.33), after stratification by PS decile (aHR 1.08; 95\% CI 0.39-3.00), or after matching (aHR 2.00; 95\% CI 0.37-10.92). 
Table 4 Baseline characteristics of new and non-new users from Truven MarketScan for the stroke outcome analysis

\begin{tabular}{|c|c|c|c|c|}
\hline Baseline patient characteristics & $\begin{array}{l}\text { Pre-matching } \\
\text { mirabegron } \\
\text { Mean }(\mathrm{SD})^{\mathrm{a}}\end{array}$ & $\begin{array}{l}\text { Pre-matching } \\
\text { oxybutynin } \\
\text { Mean }(\mathrm{SD})^{\mathrm{a}}\end{array}$ & $\begin{array}{l}\text { Post-matching } \\
\text { mirabegron } \\
\text { Mean (SD) }\end{array}$ & $\begin{array}{l}\text { Post-matching } \\
\text { oxybutynin } \\
\text { Mean }(\mathrm{SD})^{\mathrm{a}}\end{array}$ \\
\hline New users & $(N=17,138)$ & $(N=63,835)$ & $(N=15,973)$ & $(N=15,973)$ \\
\hline Age at index date & $64.6(15.2)$ & $59.6(16.2)$ & $64.3(15.3)$ & $62.8(15.9)$ \\
\hline \multicolumn{5}{|l|}{ Gender $(N[\%])$} \\
\hline Male & $5477(32.0 \%)$ & $23,552(36.9 \%)$ & $5065(31.7 \%)$ & $5080(31.8 \%)$ \\
\hline Female & $11,661(68.0 \%)$ & $40,283(63.1 \%)$ & $10,908(68.3 \%)$ & $10,893(68.2 \%)$ \\
\hline $\begin{array}{l}\text { Charlson-Elixhauser co-morbidity } \\
\text { score }\end{array}$ & $0.6(1.5)$ & $0.7(1.8)$ & $0.6(1.5)$ & $0.6(1.5)$ \\
\hline Inpatient hospitalizations & $0.4(2.1)$ & $0.6(2.5)$ & $0.4(2.1)$ & $0.4(1.7)$ \\
\hline Emergency department visits & $0.2(0.7)$ & $0.4(0.9)$ & $0.2(0.7)$ & $0.2(0.6)$ \\
\hline Non-acute institutional stays & $0.2(2.3)$ & $0.3(3.6)$ & $0.2(2.3)$ & $0.2(2.5)$ \\
\hline Ambulatory visits & $2.4(4.3)$ & $2.9(5.0)$ & $2.4(4.4)$ & $2.4(4.0)$ \\
\hline Other ambulatory events & $11.4(10.0)$ & $8.6(9.1)$ & $10.9(9.6)$ & $11.0(11.0)$ \\
\hline Unique prescriptions & $18.7(15.6)$ & $16.3(15.0)$ & $18.4(15.5)$ & $18.6(15.9)$ \\
\hline Unique generics & $8.6(5.5)$ & $7.4(5.2)$ & $8.4(5.4)$ & $8.4(5.6)$ \\
\hline Non-new users & $(N=15,173)$ & $(N=11,314)$ & $(N=8103)$ & $(N=8103)$ \\
\hline Age at index date & $66.3(14.5)$ & $65.3(15.3)$ & $66.2(14.7)$ & $64.8(14.9)$ \\
\hline \multicolumn{5}{|l|}{ Gender $(N[\%])$} \\
\hline Male & $4083(26.9 \%)$ & $2707(23.9 \%)$ & $2052(25.3 \%)$ & $2052(25.3 \%)$ \\
\hline Female & $11,090(73.1 \%)$ & $8607(76.1 \%)$ & $6051(74.7 \%)$ & $6051(74.7 \%)$ \\
\hline $\begin{array}{l}\text { Charlson-Elixhauser co-morbidity } \\
\text { score }\end{array}$ & 0.7 (1.6) & $0.8(1.9)$ & $0.7(1.7)$ & $0.6(1.7)$ \\
\hline Inpatient hospitalizations & $0.4(1.8)$ & $0.8(3.3)$ & $0.5(2.1)$ & $0.5(2.1)$ \\
\hline Emergency department visits & $0.3(0.7)$ & $0.4(0.9)$ & $0.3(0.8)$ & $0.3(0.7)$ \\
\hline Non-acute institutional stays & $0.3(2.6)$ & $0.4(2.9)$ & $0.3(2.7)$ & $0.3(2.2)$ \\
\hline Ambulatory visits & $2.8(5.1)$ & $2.9(4.9)$ & $2.8(5.2)$ & $2.7(4.8)$ \\
\hline Other ambulatory events & $12.1(10.4)$ & $10.3(10.1)$ & $10.7(9.8)$ & $10.6(10.0)$ \\
\hline Unique prescriptions & $24.2(17.5)$ & $23.3(17.9)$ & $23.5(17.2)$ & $23.3(17.6)$ \\
\hline Unique generics & $10.5(5.7)$ & $9.9(5.6)$ & $10.0(5.5)$ & $10.0(5.6)$ \\
\hline
\end{tabular}

$N A$ not applicable (propensity score matching model did not converge, $S D$ standard deviation

${ }^{a}$ Data appears as mean (SD) except for gender data, which appears as $N(\%)$

During follow-up, the IR of stroke was 6.3/1000 PY among new mirabegron users and 9.5/1000 PY among new oxybutynin users in PharMetrics, with a HR in the unmatched groups of 0.66 (95\% CI 0.39-1.13). The aHR after stratification of PS decile was $0.62 \quad(95 \%$ CI 0.34-1.13); the model in the PS-matched groups did not converge.

Among non-new users in PharMetrics, the IRs of stroke were 5.1/1000 PY for mirabegron and 6.3/1000 PY for oxybutynin. No statistically significant association was observed between mirabegron and stroke in the unmatched cohort model (HR 0.67; 95\% CI 0.33-1.36), after stratification by PS decile (aHR $0.69 ; 95 \%$ CI $0.29,1.61$ ), or following PS matching (aHR 0.25; 95\% CI 0.03-2.24).

Among new users in Truven MarketScan for the AMI outcome analysis, the mean length of mirabegron exposure was 92 days compared to 52 days on oxybutynin (data not shown). Incidence rates were 3.7/1000 PY for mirabegron and 6.8/1000 PY for oxybutynin, while model results from unmatched treatment groups (HR 0.48; 95\% CI 0.28-0.83), and after PS decile stratification (aHR 0.54; 95\% CI 0.30-0.98) were similar. After PS matching, no statistically significant association was observed (aHR 0.57 ; 95\% CI 0.17-1.95).

Among non-new users in MarketScan, the IR of AMI for mirabegron was 6.1/1000 PY and 8.3/1000 PY for oxybutynin. No statistically significant association was observed between non-new mirabegron use and AMI in the unmatched cohorts (HR 0.61; 95\% CI 0.36-1.05) after stratification by PS decile (aHR $0.71 ; 95 \%$ CI $0.38-1.29$ ) or after matching (aHR 0.80; 95\% CI 0.32-2.03). 
Table 5 Outcomes analyses for new and non-new users from IMS PharMetrics and Truven MarketScan

\begin{tabular}{|c|c|c|c|c|c|}
\hline & $\begin{array}{l}\text { Mirabegron } \\
\text { IR/1000 PY }\end{array}$ & $\begin{array}{l}\text { Oxybutynin } \\
\text { IR/1000 PY }\end{array}$ & $\begin{array}{l}\text { Unadjusted } \\
\mathrm{HR}^{\mathrm{b}}(95 \% \mathrm{CI})\end{array}$ & $\begin{array}{l}\text { Adjusted } \mathrm{HR}^{\mathrm{b}}(95 \% \mathrm{CI}) \\
\text { Stratified by PS decile }\end{array}$ & $\begin{array}{l}\text { Adjusted } \mathrm{HR}^{\mathrm{b}}(95 \% \mathrm{CI}) \\
\text { Matching on PS }\end{array}$ \\
\hline \multicolumn{6}{|c|}{ IMS PharMetrics, new users } \\
\hline AMI & 4.4 & 6.5 & $0.68(0.36-1.28)$ & $0.67(0.33-1.37)$ & NA \\
\hline Stroke & 6.3 & 9.5 & $0.66(0.39-1.13)$ & $0.62(0.34-1.13)$ & NA \\
\hline \multicolumn{6}{|c|}{ IMS PharMetrics, non-new users } \\
\hline AMI & 5.8 & 2.7 & $0.95(0.38-2.33)$ & $1.08(0.39-3.00)$ & $2.00(0.37-10.92)$ \\
\hline Stroke & 5.1 & 6.3 & $0.67(0.33-1.36)$ & $0.69(0.29-1.61)$ & $0.25(0.03-2.24)$ \\
\hline \multicolumn{6}{|c|}{ Truven MarketScan, new users } \\
\hline AMI & 3.7 & 6.8 & $0.48(0.28-0.83)$ & $0.54(0.30-0.98)$ & $0.57(0.17-1.95)$ \\
\hline Stroke & 5.3 & 8.4 & $0.63(0.41-0.98)$ & $0.65(0.40-1.06)$ & $0.69(0.30-1.62)$ \\
\hline \multicolumn{6}{|c|}{ Truven MarketScan, non-new users } \\
\hline AMI & 6.1 & 8.3 & $0.61(0.36-1.05)$ & $0.71(0.38-1.29)$ & $0.80(0.32-2.03)$ \\
\hline Stroke & 14.7 & 8.8 & $1.20(0.78-1.84)$ & $1.51(0.93-2.44)$ & $0.92(0.40-2.08)$ \\
\hline
\end{tabular}

$A M I$ acute myocardial infarction, $C I$ confidence interval, $H R$ hazard ratio, $I R$ incidence rate, $N A$ not applicable, $P S$ propensity score, $P Y$ personyears

${ }^{a}$ Incidence rates are calculated from the PS-matched treatment groups; when the PS-matching model did not converge, incidence rates are calculated from the unmatched treatment groups stratified by propensity score

${ }^{\mathrm{b}} \mathrm{HRs}$ are associated with mirabegron use, with oxybutynin use as the reference

Among new users in MarketScan, IRs of stroke varied from 5.3/1000 PY among mirabegron users to 8.4/1000 PY among oxybutynin users. In the unmatched new users, the HR was 0.63 (95\% CI 0.41-0.98) in the stroke analysis. The aHR after PS decile stratification $(0.65 ; 95 \%$ CI $0.40-1.06)$ was similar to the model of matched groups (aHR 0.69; 95\% CI 0.30-1.62), although the CI was broader.

Among non-new users in MarketScan, IRs for stroke were 14.7/1000 PY in mirabegron users and 8.8/1000 PY in oxybutynin users. No statistically significant association was observed between non-new use of mirabegron and stroke, in unmatched treatment groups (HR 1.20; 95\% CI $0.78-1.84$ ), after stratification by PS decile (aHR $1.51 ; 95 \%$ CI 0.93-2.44), and after matching (aHR 0.92; 95\% CI $0.40-2.08)$.

\section{Discussion}

The present study assessed two large US administrative claims databases from 2012-2015 and did not identify a statistically significant increased risk of AMI or stroke among new or non-new mirabegron users compared to oxybutynin users. These findings were consistent prior to and after matching (when the model converged) on PS created from demographic and clinical characteristics, as well as healthcare resource utilization data. To our knowledge, this is the first published attempt at replicating an M-S safety study using the publicly-available
CDM specifications, study protocol, and PROMPT 2 module, using data sources other than those participating in M-S.

The FDA M-S reports on mirabegron published in September 2016 similarly found no increased risk of AMI or stroke among mirabegron users compared to oxybutynin users [12]. For instance, in the new user analysis of primary diagnoses of AMI, 4465 mirabegron users and 4464 oxybutynin users were matched [12]. The aHR for matched treatment groups was 1.00 (95\% CI 0.14-7.10), while the wide confidence intervals reflected relatively few outcomes observed during the study period (five cases of AMI among mirabegron users vs. three among oxybutynin users) [12]. In the matched analysis of primary diagnoses of stroke, an aHR of 0.80 (95\% CI 0.21-2.98) was reported [12]. Published studies to date have reported no association of mirabegron use and increased AMI and/or stroke risk $[13,14]$.

Strengths of the FDA's M-S program include the use of a CDM, standardized cohort selection, and analysis modules that are publicly available and used by the M-S data partners. As one of the goals of this analysis was to replicate the methods of the FDA's M-S study, only a minor necessary deviation from the M-S CDM was made to identify unique patient visits. This overall consistency makes the results comparable to the findings reported by the FDA's M-S study of mirabegron, even though different analysis datasets were used. The methods described here may be applied by other researchers who wish to replicate a Mini-Sentinel study in other databases. 
Some known limitations inherent to administrative claims databases must also be noted. Claims diagnoses represent justifications for billing and may not always accurately reflect patients' medical conditions. Variables that might be found in electronic health record data, such as alcohol consumption and body mass index, are not available in administrative claims databases; however, all variables specified in the M-S protocol could be coded in the datasets used. Confounders of outcomes related to the decision to treat with mirabegron versus oxybutynin may exist despite the use of PS matching.

A pharmacy claim indicates the availability of a medication to a patient, not actual use of that medication. Therefore, details of medication dispensing only approximate actual treatment patterns. Health care received outside of the health care plan, such as use of over-the-counter medications, do not appear in the claims data. Claims databases do not capture the reasons for failure to refill medications.

The databases used in this study are large commercial administrative claims databases, and they are considered to be generalizable to the US population with access to commercial health insurance. It is likely that there is some patient overlap between the PharMetrics and MarketScan databases, and it is possible that some overlap could be present with the M-S data. As patients are anonymized, however, the amount of any overlap cannot be determined and this information is not disclosed by the data vendors. Lastly, although use of the PROMPT 2 module was specified in the Mini-Sentinel protocol for mirabegron, this module has since been replaced by the Cohort Identification and Descriptive Analysis (CIDA) + Propensity Score (PS) tool; analyses conducted using the updated CIDA + PS tool may differ from those presented here.

\section{Conclusions}

This study of two large US administrative claims databases did not detect a statistically significant increased risk of either AMI or stroke among new or non-new users of mirabegron compared with oxybutynin users. These findings are consistent with both the FDA's Mini-Sentinel safety study of mirabegron and other published literature. The replication methods described here may be considered for other therapies, outcomes, and databases of interest to researchers.

Acknowledgements The authors thank Noah Jamie Robinson of Astellas for his contributions in the review and editing of this manuscript. They also thank Janet Dooley of the Evidera Production team for her assistance in preparing the manuscript for submission.
Presentations An abstract associated with this study was accepted for an oral/podium presentation at the 33rd International Conference on Pharmacoepidemiology and Therapeutic Risk Management, 26-30 August 2017, in Montreal, Canada.

\section{Compliance with Ethical Standards}

Funding information This study and medical writing support for this manuscript was funded by Astellas Inc. Astellas Pharma US, Inc. of Northbrook, Illinois markets and distributes Myrbetriq ${ }^{\circledR}$ (mirabegron). Evidera received funding from Astellas, Inc. for their work on the project and manuscript.

Authors' conflicts of interest Kwame Appenteng and Milbhor D'Silva are employees and minor shareholders of Astellas Pharma US, Inc. of Northbrook, Illinois. Jason Simeone, Beth Nordstrom, and Samuel Huse are salaried employees of Evidera, a research/consulting firm for the biopharmaceutical industry. As such, they are precluded from receiving payment or honoraria directly from any of Evidera's clients.

Ethical approval/informed consent For this type of study, formal consent is not required.

Open Access This article is distributed under the terms of the Creative Commons Attribution-NonCommercial 4.0 International License (http://creativecommons.org/licenses/by-nc/4.0/), which permits any noncommercial use, distribution, and reproduction in any medium, provided you give appropriate credit to the original author(s) and the source, provide a link to the Creative Commons license, and indicate if changes were made.

\section{References}

1. Chen GD, Lin TL, Hu SW, et al. Prevalence and correlation of urinary incontinence and overactive bladder in Taiwanese women. Neurourol Urodyn. 2003;22(2):109-17. https://doi.org/ 10.1002/nau.10010.

2. Cheung WW, Blank W, Borawski D, et al. Prevalence of overactive bladder, its under-diagnosis, and risk factors in a male urologic veterans population. Int J Med Sci. 2010;7(6):391-4.

3. Cheung WW, Khan NH, Choi KK, et al. Prevalence, evaluation and management of overactive bladder in primary care. BMC Fam Pract. 2009;10:8. https://doi.org/10.1186/1471-2296-10-8.

4. Corcos J, Schick E. Prevalence of overactive bladder and incontinence in Canada. Can J Urol. 2004;11(3):2278-84.

5. Astellas Pharma US Inc. Myrbetriq ${ }^{\circledR}$ Full Prescribing Information. Astellas Pharma US, Inc., Northbrook, Illinois. Revised: 8/2016. https://www.us.astellas.com/docs/Myrbetriq_WPI.pdf. Accessed 28 Feb 2017.

6. MacMahon S, Peto R, Cutler J, et al. Blood pressure, stroke, and coronary heart disease. Part 1 , Prolonged differences in blood pressure: prospective observational studies corrected for the regression dilution bias. Lancet. 1990;335(8692):765-74.

7. National Heart Lung and Blood Institute (US). The Seventh Report of the Joint National Committee on Prevention, Detection, Evaluation, and Treatment of High Blood Pressure. Report Number 04-5230. Bethesda (MD). 2004. http://www.ncbi.nlm. nih.gov/pubmed/20821851. Accessed 12 Jan 2017.

8. Make B, Dutro MP, Paulose-Ram R, et al. Undertreatment of COPD: a retrospective analysis of US managed care and 
Medicare patients. Int J Chron Obstruct Pulmon Dis. 2012;7:1-9. https://doi.org/10.2147/COPD.S27032.

9. Hawkins NM, Petrie MC, Jhund PS, et al. Heart failure and chronic obstructive pulmonary disease: diagnostic pitfalls and epidemiology. Eur J Heart Fail. 2009;11(2):130-9. https://doi. org/10.1093/eurjhf/hfn013.

10. Leonard CE, Reichman ME, Toh D, et al. Mini-Sentinel Prospective Surveillance Plan. Prospective Routine Observational Monitoring of Mirabegron: Version 2.0. Sentinel Coordinating Center. 2014. http://www.mini-sentinel.org/work_products/ Assessments/Mini-Sentinel_PROMPT_Mirabegron-SurveillancePlan.pdf. Accessed 13 Nov 2015.

11. Platt R, Archdeacon P, Bell C, et al. Mini-Sentinel Methods; Prospective Routine Observational Monitoring Program Tools (PROMPT) User's Guide. 2014. https://www.sentinelinitiative. org/sites/default/files/Methods/Mini-Sentinel_PROMPT_UsersGuide_0.pdf. Accessed 10 April 2017.
12. Leonard CE, Reichman ME, Toh D, et al. Mini-Sentinel Prospective Surveillance Plan: Prospective Routine Observational Monitoring of Mirabegron. Sentinel Coordinating Center. 2016. https://www.sentinelinitiative.org/sites/default/files/Drugs/ Assessments/Mini-Sentinel_Modular-Program-Report_to12 prompt_mira_mp13r_wp01_nsdp_v01_1-4.pdf. Accessed 12 Jan 2017.

13. Robinson D, Thiagamoorthy G, Cardozo L. A drug safety evaluation of mirabegron in the management of overactive bladder. Expert Opin Drug Saf. 2016;15(5):689-96. https://doi.org/10. 1517/14740338.2016.1165663.

14. Rosa GM, Ferrero S, Nitti VW, et al. Cardiovascular safety of beta3-adrenoceptor agonists for the treatment of patients with overactive bladder syndrome. Eur Urol. 2016;69(2):311-23. https://doi.org/10.1016/j.eururo.2015.09.007. 